\title{
JAARCIJFERS VAN SURINAME VI
}

DOOR

L. C. PREY.

OPENBAAR ONDERWIJS

\begin{tabular}{|c|c|c|c|c|c|c|c|c|c|c|c|c|c|c|c|c|c|c|c|c|c|c|c|c|c|c|c|c|c|c|c|}
\hline \multirow{4}{*}{ 总 } & \multicolumn{8}{|c|}{ Aantal scholen } & \multirow{2}{*}{\multicolumn{3}{|c|}{$\begin{array}{c}\text { Aantal ge- } \\
\text { exami- } \\
\text { neerde on- } \\
\text { derwijzers }\end{array}$}} & \multirow{2}{*}{\multicolumn{17}{|c|}{ Aantal leerlingen }} & \multirow{3}{*}{\multicolumn{3}{|c|}{$\begin{array}{l}\text { Geheele ge- } \\
\text { west (zonder } \\
\text { Boschland- } \\
\text { scholen) }\end{array}$}} \\
\hline & \multicolumn{4}{|c|}{ Paramaribo } & \multicolumn{3}{|c|}{ Districten } & \multirow{3}{*}{ 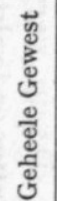 } & & & & & & & & & & & & & & & & & & & & & & & \\
\hline & \multirow{2}{*}{ ن } & \multirow{2}{*}{$\begin{array}{l}0 \\
\dot{0} \\
\dot{0}\end{array}$} & \multirow{2}{*}{$\begin{array}{l}\dot{0} \\
\dot{j} \\
\dot{p} \\
\dot{z}\end{array}$} & \multirow{2}{*}{ స్త్ } & \multirow{2}{*}{$\begin{array}{l}\dot{0} \\
\dot{j} \\
\dot{0}\end{array}$} & \multirow{2}{*}{$\begin{array}{l}0 \\
\dot{0} \\
\dot{j} \\
\dot{n}\end{array}$} & \multirow{2}{*}{ 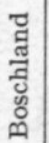 } & & \multirow{2}{*}{\multicolumn{2}{|c|}{ 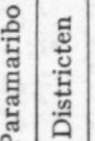 }} & \multirow{2}{*}{ స్తึ } & \multicolumn{3}{|c|}{ G.L.O. } & \multicolumn{3}{|c|}{ U.L.O. } & \multicolumn{3}{|c|}{ M.U.L.O. } & \multicolumn{3}{|c|}{ Totaal } & \multicolumn{3}{|c|}{ G. en B.L.O. ${ }^{1}$ ) } & \multicolumn{2}{|c|}{$\begin{array}{l}\text { Bosch- } \\
\text { land }\end{array}$} & & & \\
\hline & & & & & & & & & & & & j. & $\mathrm{m}$. & t. & j. & m. & t. & j. & $\mathrm{m}$. & t. & j. & $\mathrm{m}$. & t. & j. & $\mathrm{m}$. & t. & j. m. & t. & j. & m. & t. \\
\hline
\end{tabular}

\begin{tabular}{r|r|r|r|r|r|l|l|l|}
\hline 1920 & 5 & 2 & - & 7 & 26 & - & - & 33 \\
1921 & 5 & 2 & - & 7 & 28 & - & - & 35 \\
1922 & 5 & 2 & - & 7 & 28 & - & - & 35 \\
1923 & 5 & 2 & - & 7 & 28 & - & - & 35 \\
1924 & 5 & 2 & - & 7 & 28 & - & - & 35 \\
1925 & 5 & 1 & 1 & 7 & 28 & - & - & 35 \\
1926 & 5 & 1 & 1 & 7 & 28 & - & - & 35 \\
1927 & 4 & 1 & 1 & 6 & 35 & - & - & 41 \\
1928 & 4 & 1 & 1 & 6 & 35 & - & - & 41 \\
1929 & 4 & 1 & 1 & 6 & 35 & - & - & 41 \\
1930 & 4 & 1 & 1 & 6 & 26 & 9 & - & 41 \\
1931 & 5 & 1 & 1 & 7 & 26 & 9 & - & 42 \\
1932 & 5 & 1 & 1 & 7 & 4 & 31 & - & 42 \\
1933 & 5 & 1 & 1 & 7 & 4 & 32 & - & 43 \\
1904 & 5 & 1 & 1 & 7 & 4 & 32 & - & 43 \\
1935 & 5 & 1 & 1 & 7 & 4 & 32 & - & 43 \\
1936 & 5 & 1 & 1 & 7 & 3 & 33 & - & 43 \\
1937 & 5 & 1 & 1 & 7 & 3 & 33 & - & 43
\end{tabular}

\begin{tabular}{l|l|l|l|l|l|l|l|l|l|l|l|l|l|l|l|l|l|l|l|l|l|l|l|l|}
98 & 48 & 146 & 788 & 874 & 1662 & 395 & 348 & 743 & - & - & - & 1183 & 1222 & 2405 & 1379 & 547 & 1926 & — & — 2562 & 1769 & 4331
\end{tabular}

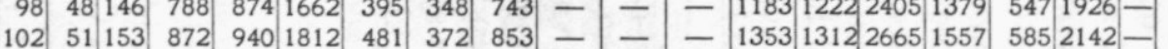
\begin{tabular}{l|l|l|l|l|l|l|l|l|l|l|l}
96 & 52 & 148 & 938 & 1014 & 1952 & 489 & 379 & 868 & -
\end{tabular} \begin{tabular}{l|l|l|l|l|l|l|l|l|l}
88 & 58 & 146 & 889 & 979 & 1868 & 496 & 463 & 959 & -
\end{tabular}

\begin{tabular}{l|l|l|l|l|l|l|l|l|l}
82 & 61 & 143 & 743 & 807 & 1550 & 524 & 480 & 1004 & - \\
\hline
\end{tabular}

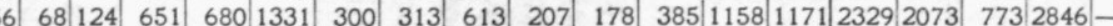
\begin{tabular}{l|l|l|l|l|l|l|l|l|l|l|l|l|l|l|l|l|l|l|l|}
59 & 67 & 126 & 662 & 649 & 1311 & 316 & 317 & 633 & 211 & 167 & 378 & 1189 & 1133 & 2322 & 2063 & 883 & 2946 & -
\end{tabular} \begin{tabular}{l|l|l|l|l|l|l|l|l|l|l|l|l|l|l|l|l|l|}
58 & 79 & 137 & 660 & 675 & 1335 & 296 & 294 & 590 & 209 & 160 & 369 & 1165 & 1129 & 2294 & 2834 & 1237 & 4071 \\
\hline
\end{tabular}

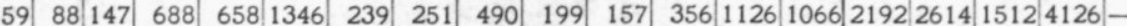
\begin{tabular}{|l|l|l|l|l|l|l|l|l|l|l|l|l|l|l|l|l|l|}
57 & 87 & 144 & 714 & 662 & 1376 & 261 & 261 & 522 & 195 & 138 & 333 & 1170 & 1061 & 2231 & 2757 & 1734 & 4491
\end{tabular}

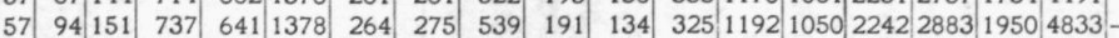

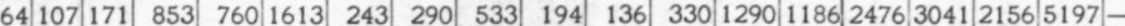
\begin{tabular}{l|l|l|l|l|l|l|l|l|l|l|l|l|l|l|l|l|l|}
69 & 78 & 147 & 804 & 711 & 1515 & 235 & 268 & 503 & 196 & 130 & 326 & 1235 & 1109 & 2344 & 3119 & 2168 & 5287 \\
\hline
\end{tabular} \begin{tabular}{ll|l|l|l|l|l|l|l|l|l|l|l|l|l|l|l|l|l|l|}
65 & 90 & 155 & 854 & 731 & 1585 & 238 & 263 & 501 & 183 & 138 & 321 & 1275 & 1132 & 2407 & 3268 & 2326 & 5594 & -
\end{tabular}

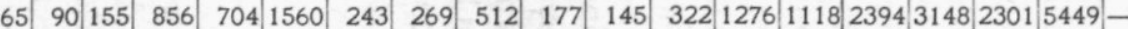
\begin{tabular}{|l|l|l|l|l|l|l|l|l|l|l|l|l|l|l|l|l|l}
64 & 81 & 145 & 794 & 646 & 1440 & 267 & 258 & 525 & 175 & 146 & 321 & 1236 & 1050 & 2286 & 3190 & 2325 & 5515
\end{tabular} \begin{tabular}{|l|l|l|l|l|l|l|l|l|l|l|l|l|l|l|l|l|l|l|}
63 & 85 & 148 & 786 & 638 & 1424 & 277 & 246 & 523 & 183 & 146 & 329 & 1246 & 1030 & 2276 & 3238 & 2374 & 5612 & -
\end{tabular}

1) B.L.O. = beperkt lager onderwijs; in 1930 had de eerste omzetting van G.L.O.-scholen in B.L.O.-scholen plaats. $5284-1-425632247490$ (- 
BIJZONDER ONDERWIJS

SCHOLEN DER EVANGELISCHE BROEDERGEMEENTE

\begin{tabular}{|c|c|c|c|c|c|c|c|c|c|c|c|c|c|c|c|c|c|c|c|c|c|c|c|c|c|c|c|c|c|c|c|c|}
\hline \multirow{4}{*}{ 㺃 } & \multicolumn{8}{|c|}{ Aantal scholen } & \multirow{2}{*}{\multicolumn{3}{|c|}{$\begin{array}{c}\text { Aantal ge- } \\
\text { examineer- } \\
\text { de onder- } \\
\text { wijzers } \\
\end{array}$}} & \multicolumn{18}{|c|}{ Aantal leerlingen } & \multirow{3}{*}{\multicolumn{3}{|c|}{$\begin{array}{l}\text { Geheele gewest } \\
\text { (zonder Bosch- } \\
\text { landscholen) }\end{array}$}} \\
\hline & \multicolumn{4}{|c|}{ Paramaribo } & \multicolumn{3}{|c|}{ Districten } & \multirow{3}{*}{ 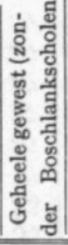 } & & & & \multicolumn{12}{|c|}{ Paramaribo } & \multicolumn{6}{|c|}{ Districten } & & & \\
\hline & \multirow{2}{*}{ 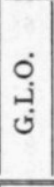 } & \multirow{2}{*}{$\begin{array}{c}\dot{0} \\
\dot{j} \\
\dot{0}\end{array}$} & \multirow{2}{*}{$\begin{array}{l}\dot{0} \\
\dot{\mu} \\
\dot{2} \\
\dot{2} \\
\dot{\Sigma}\end{array}$} & \multirow{2}{*}{ సี } & \multirow{2}{*}{$\begin{array}{l}\dot{0} \\
\dot{j} \\
ن\end{array}$} & \multirow{2}{*}{$\begin{array}{l}\approx \\
\dot{0} \\
\dot{\leftrightarrow}\end{array}$} & \multirow{2}{*}{ 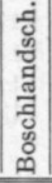 } & & \multirow{2}{*}{ 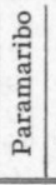 } & \multirow{2}{*}{ 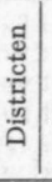 } & \multirow{2}{*}{ đ్త్ } & \multicolumn{3}{|c|}{ G.L.O. ${ }^{2}$ ) } & \multicolumn{3}{|c|}{ U.L.O. } & M. & U.L.C & & & Totaal & & G. en & B.L. & $\left.{ }^{1}\right)$ & $\begin{array}{l}\text { Bos } \\
\text { sch }\end{array}$ & $\begin{array}{l}\text { schla } \\
\text { holen }\end{array}$ & $\begin{array}{l}\text { and- } \\
\left.n^{3}\right)\end{array}$ & & & \\
\hline & & & & & & & & & & & & j. & m. & t. & j. & m. & t. & j. & $\mathrm{m}$. & t. & j. & m. & t. & j. & $\mathrm{m}$. & t. & j. & m. & t. & j. & m. & t. \\
\hline 1920 & 7 & 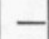 & - & 7 & 13 & - & 12 & 20 & 47 & 28 & 75 & 734 & 630 & 1364 & & & & & & & 734 & 630 & 1364 & 655 & 474 & 1129 & - & - & - & -1389 & 1104 & 2493 \\
\hline 1921 & 7 & - & 一 & 7 & 14 & - & 9 & 21 & 49 & 30 & 79 & 802 & 637 & 1439 & & & & & & & 802 & 637 & 1439 & 675 & 467 & 1142 & - & 一 & - & 1477 & 1104 & 2581 \\
\hline 1922 & 7 & - & - & 7 & 14 & - & 9 & 21 & 52 & 32 & 84 & 995 & 781 & 1776 & & & & & & & 995 & 781 & 1776 & 782 & 524 & 1306 & - & - & - & -1777 & 1305 & 3082 \\
\hline 1923 & 6 & 1 & - & 7 & 17 & - & 9 & 24 & 56 & 35 & 91 & 1127 & 886 & 2013 & & & & & & & 1127 & 886 & 2013 & 897 & 595 & 1492 & - & - & - & 2024 & 1481 & 3505 \\
\hline 1924 & 6 & 1 & - & 7 & 19 & - & 11 & 26 & 56 & 40 & 96 & 1101 & 910 & 2011 & & & & & & & 1101 & 910 & 2011 & 1056 & 659 & 1715 & - & - & - & 2157 & 1569 & 3726 \\
\hline 1925 & 6 & 1 & - & 7 & 19 & - & 10 & 26 & 44 & 47 & 91 & 993 & 908 & 1901 & & & & & & & 993 & 908 & 1901 & 1128 & 750 & 1878 & - & - & - & 2121 & 1658 & 3779 \\
\hline 1926 & 6 & 1 & - & 7 & 19 & - & 10 & 26 & 43 & 43 & 86 & 1014 & 983 & 1997 & & & & & & & 1014 & 983 & 1997 & 1184 & 805 & 1989 & - & - & - & 2198 & 1788 & 3986 \\
\hline 1927 & 6 & 1 & - & 7 & 20 & - & 12 & 27 & 47 & 46 & 93 & 755 & 814 & 1569 & 354 & 252 & 606 & & & & 1109 & 1066 & 2175 & 1117 & 816 & 1933 & - & - & - & 2220 & 1882 & 4102 \\
\hline 1928 & 6 & 1 & 1 & 8 & 21 & - & 12 & 28 & 54 & 45 & 99 & 638 & 732 & 1370 & 333 & 263 & 596 & 158 & 103 & 261 & 1129 & 1098 & 2227 & 1064 & 761 & 1825 & - & - & - & 2193 & 1859 & 4052 \\
\hline 1929 & 6 & 1 & 1 & 8 & 22 & - & 12 & 30 & 64 & 42 & 106 & 795 & 864 & 1659 & 307 & 223 & 530 & 135 & 95 & 230 & 1237 & 1182 & 2419 & 1086 & 827 & 1913 & - & - & - & 2323 & 2009 & 4332 \\
\hline 1930 & 7 & 1 & 1 & 9 & 19 & 5 & 15 & 33 & 63 & 44 & 122 & 874 & 924 & 1798 & 300 & 209 & 509 & 128 & 89 & 217 & 1302 & 1222 & 2524 & 1169 & 907 & 2076 & - & - & - & 2695 & 2338 & 5033 \\
\hline 1931 & 7 & 1 & 1 & 9 & 19 & 4 & 16 & 32 & 70 & 53 & 123 & 928 & 971 & 1899 & 287 & 212 & 499 & 136 & 99 & 235 & 1351 & 1282 & 2633 & 1165 & 952 & 2117 & $1-$ & - & - & 2516 & 2234 & 4750 \\
\hline 1932 & 7 & 1 & 1 & 9 & 5 & 19 & 14 & 33 & 70 & 46 & 116 & 923 & 937 & 1860 & 241 & 202 & 443 & 131 & 95 & 226 & 1295 & 1234 & 2529 & 1222 & 949 & 2171 & - & - & - & 2517 & 2183 & 4700 \\
\hline 1933 & 7 & 1 & 1 & 9 & 5 & 19 & 14 & 33 & 70 & 46 & 116 & 906 & 987 & 1893 & 215 & 190 & 405 & 124 & 103 & 227 & 1245 & 1280 & 2525 & 1287 & 922 & 22092 & 244 & 239 & 483 & 2532 & 2202 & 4734 \\
\hline 1934 & 7 & 1 & 1 & 9 & 5 & 19 & 14 & 33 & 70 & 48 & 118 & 916 & 991 & 1907 & 222 & 193 & 415 & 107 & 91 & 198 & 1345 & 1275 & 2520 & 1300 & 970 & 2270 & 226 & 229 & 455 & 2545 & 2245 & 4790 \\
\hline 1935 & 7 & 1 & 1 & 9 & 5 & 19 & 14 & 33 & 60 & 43 & 103 & 996 & 1025 & 2021 & 205 & 175 & 380 & 105 & 89 & 194 & 1306 & 1289 & 2595 & 1303 & 1023 & 2326 & 222 & 217 & 439 & 2609 & 2312 & 4921 \\
\hline 1936 & 7 & 1 & 1 & 9 & 4 & 20 & 15 & 33 & 64 & 48 & 112 & 1069 & 1133 & 2202 & 201 & 163 & 364 & 100 & 94 & 194 & 1370 & 1390 & 2760 & 1379 & 1073 & 2452 & 254 & 221 & 475 & 2749 & 2463 & 5212 \\
\hline 1937 & 7 & 1 & 1 & 9 & 5 & 19 & 14 & 33 & 68 & 52 & 120 & 1094 & 1159 & 2253 & 210 & 149 & 359 & 92 & 85 & 177 & $|1396|$ & | 1393 & 2789 & $|1423|$ & 1128 & $|2551|^{2}$ & $|250|$ & $|211|$ & 461 & $\mid 2819$ & 2521 & 5340 \\
\hline
\end{tabular}

$\left.{ }^{1}\right)$ B.L.O. = beperkt lager onderwijs; in 1930 had de eerste omzetting van G.L.O.-scholen in B.L.O.-scholen plaats.

2) Voor de jaren 1920 t/m 1926 zijn de cijfers voor G.L.O. en U.L.O. samengevoegd.

3) Over de jaren 1920 t/m 1932 zijn geen cijfers bekend. 
BIJZONDER ONDERWIJS

SCHOLEN DER ROOMSCH-KATHOLIEKE GEMEENTE

\begin{tabular}{|c|c|c|c|c|c|c|c|c|c|c|c|c|c|c|c|c|c|c|c|c|c|c|c|c|c|c|c|c|c|c|c|c|}
\hline \multirow{4}{*}{ 总 } & \multicolumn{8}{|c|}{ Aantal scholen } & \multirow{2}{*}{\multicolumn{3}{|c|}{$\begin{array}{c}\text { Aantal ge- } \\
\text { examineer- } \\
\text { de onder- } \\
\text { wijzers } \\
\end{array}$}} & \multicolumn{18}{|c|}{ Aantal leerlingen } & \multirow{3}{*}{\multicolumn{3}{|c|}{$\begin{array}{l}\text { Geheele Gewest } \\
\text { (zonder Bosch- } \\
\text { landscholen) }\end{array}$}} \\
\hline & \multicolumn{4}{|c|}{ Paramaribo } & \multicolumn{3}{|c|}{ Districten } & 这 옹 & & & & \multicolumn{12}{|c|}{ Paramaribo ${ }^{2}$ ) } & \multicolumn{6}{|c|}{ Districten } & & & \\
\hline & \multirow{2}{*}{$\begin{array}{c}0 \\
j \\
\dot{j}\end{array}$} & \multirow{2}{*}{ i. } & \multirow{2}{*}{$\begin{array}{l}0 \\
0 \\
1 \\
\dot{n} \\
\dot{2} \\
\dot{z}\end{array}$} & \multirow{2}{*}{ ঙ્刃 } & \multirow{2}{*}{ ن } & \multirow{2}{*}{$\begin{array}{l}\tilde{0} \\
\dot{0} \\
\dot{j} \\
\dot{n}\end{array}$} & \multirow{2}{*}{ 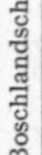 } & \multirow{2}{*}{ 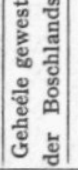 } & \multirow{2}{*}{ 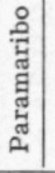 } & \multirow{2}{*}{ 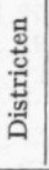 } & \multirow{2}{*}{ 丞 } & \multicolumn{3}{|c|}{ G.L.O. } & \multicolumn{3}{|c|}{ U.L.O. } & \multicolumn{3}{|c|}{ M.U.L.O. } & \multicolumn{3}{|c|}{ Totaal } & \multicolumn{3}{|c|}{ G. en B.L.O. ${ }^{1}$ ) } & \multicolumn{3}{|c|}{$\begin{array}{c}\text { Boschland } \\
\text { scholen }\end{array}$} & & & \\
\hline & & & & & & & & & & & & j. & m. & t. & j. & m. & t. & j. & m. & t. & j. & $\mathrm{m}$. & t. & j. & m. & t. & j. & $\mathrm{m}$. & t. & j. & m. & t. \\
\hline
\end{tabular}

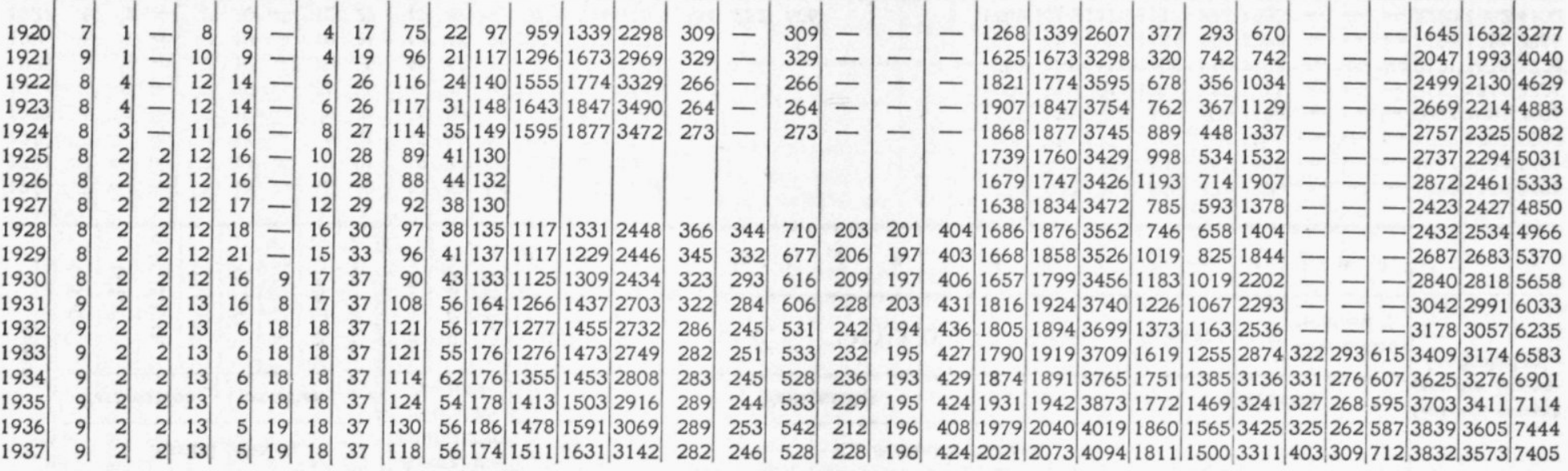

1) B.L.O. = beperkt lager onderwijs; in 1930 had de eerste omzetting van G.L.O.-scholen in B.L.O.-scholen plaats.

2) Van de jaren 1925, 1926 en 1927 ontbreken gegevens voor de verdeeling van het aantal leerlingen over G.L.O., U.L.O.O. en M.U.L.O. 
BIJZONDER ONDERWIJS

SCHOLEN DER GEMEENTE VAN GEDOOPTE CHRISTENEN

\begin{tabular}{|c|c|c|c|c|c|c|c|c|c|c|c|c|c|c|c|c|c|c|c|c|c|c|c|c|c|c|c|c|c|c|c|c|}
\hline \multirow{4}{*}{$\begin{array}{l}\text { 륩 } \\
\text { ్ㅐㅁ }\end{array}$} & \multicolumn{8}{|c|}{ Aantal scholen } & \multirow{2}{*}{\multicolumn{3}{|c|}{$\begin{array}{c}\text { Aantal ge- } \\
\text { examineer- } \\
\text { de onder- } \\
\text { wijzers }\end{array}$}} & \multicolumn{18}{|c|}{ Aantal leerlingen } & \multirow{3}{*}{\multicolumn{3}{|c|}{$-\begin{array}{l}\text { Geheele Gewest } \\
\text { (zonder Bosch- } \\
\text { landscholen). }\end{array}$}} \\
\hline & \multicolumn{4}{|c|}{ Paramaribo } & \multicolumn{3}{|c|}{ Districten } & \multirow{3}{*}{ 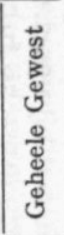 } & & & & \multicolumn{12}{|c|}{ Paramaribo } & \multicolumn{6}{|c|}{ Districten. } & & & \\
\hline & \multirow{2}{*}{ ن } & \multirow{2}{*}{$\begin{array}{l}\dot{0} \\
\dot{u} \\
\dot{p}\end{array}$} & \multirow{2}{*}{ 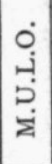 } & \multirow{2}{*}{ 胥 } & \multirow{2}{*}{ ن } & \multirow{2}{*}{$\begin{array}{l}\approx \\
\dot{0} \\
\dot{\mu} \\
\dot{m}\end{array}$} & \multirow{2}{*}{ 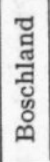 } & & \multirow{2}{*}{ 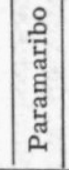 } & \multirow{2}{*}{ 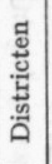 } & \multirow{2}{*}{ 졿 } & \multicolumn{3}{|c|}{ G.L.O. } & \multicolumn{3}{|c|}{ U.L.O. } & & J.L & & & otaal & & G. er & B.L & D. $\left.{ }^{1}\right)$ & Bos & chla & ind & & & \\
\hline & & & & & & & & & & & & j. & $\mathrm{m}$. & t. & j. & $\mathrm{m}$. & t. & j. & $\mathrm{m}$. & t. & j. & $\mathrm{m}$. & t. & j. & n. & t. & j. & m. & t. & j. & m. & t. \\
\hline 1920 & 1 & - & - & 1 & - & - & - & 1 & 9 & - & 9 & 103 & 152 & 255 & - & - & - & - & - & - & 103 & 152 & 255 & - & - & - & - & - & - & 103 & 152 & 255 \\
\hline 1921 & 1 & 一 & 一 & 1 & - & - & - & 1 & 9 & - & 9 & 117 & 156 & 273 & - & - & - & - & - & - & 117 & 156 & 273 & - & - & - & - & - & - & 117 & 156 & 273 \\
\hline 1922 & 1 & 一 & - & 1 & - & 7 & - & 1 & 9 & - & 9 & 118 & 172 & 290 & - & - & - & - & - & - & 118 & 172 & 290 & - & - & - & - & - & - & 118 & 172 & 290 \\
\hline 1923 & 1 & - & - & 1 & - & - & - & 1 & 9 & - & 9 & 141 & 182 & 323 & - & - & - & - & - & - & 141 & 182 & 323 & - & - & - & - & - & - & 141 & 182 & 323 \\
\hline 1924 & 1 & - & - & 1 & - & - & - & 1 & 13 & - & 13 & 171 & 216 & 387 & - & - & - & - & - & - & 171 & 216 & 387 & - & - & - & - & - & - & 171 & 216 & 387 \\
\hline 1925 & 1 & - & - & 1 & - & - & - & 1 & 7 & - & 7 & 161 & 187 & 348 & - & - & - & - & - & - & 161 & 187 & 348 & - & - & & - & - & - & 161 & 187 & 348 \\
\hline 1926 & 1 & - & - & 1 & - & - & - & 1 & 7 & - & 7 & 178 & 209 & 387 & - & - & - & - & - & - & 178 & 209 & 387 & - & - & - & - & - & - & 178 & 209 & 387 \\
\hline 1927 & 1 & - & - & 1 & - & - & - & 1 & 8 & - & 8 & 184 & 215 & 399 & - & - & - & - & - & - & 184 & 215 & 399 & - & - & - & - & - & - & 184 & 215 & 399 \\
\hline 1928 & 1 & - & - & 1 & - & - & - & 1 & 8 & - & 8 & 184 & 206 & 390 & - & - & - & - & - & - & 184 & 206 & 390 & - & - & - & - & - & - & 184 & 206 & 390 \\
\hline 1929 & 1 & - & - & 1 & - & - & - & 1 & 9 & - & 9 & 203 & 230 & 433 & - & - & - & - & - & - & 203 & 230 & 433 & - & - & - & - & - & - & 203 & 230 & 433 \\
\hline 1930 & 1 & - & - & 1 & - & - & - & 1 & 9 & - & 9 & 211 & 256 & 467 & - & - & - & - & - & - & 211 & 256 & 467 & - & - & - & - & - & - & 211 & 256 & 467 \\
\hline 1931 & 1 & - & - & 1 & - & - & - & 1 & 10 & - & 10 & 243 & 269 & 512 & - & - & - & - & - & - & 243 & 269 & 512 & - & - & - & - & - & - & 243 & 269 & 512 \\
\hline 1932 & 1 & - & - & 1 & - & - & - & 1 & 14 & - & 14 & 283 & 305 & 588 & - & - & - & - & - & - & 283 & 305 & 588 & - & - & - & - & - & - & 283 & 305 & 588 \\
\hline 1933 & 1 & - & - & 1 & - & - & - & 1 & 16 & - & 16 & 330 & 362 & 692 & - & - & - & - & - & - & 330 & 362 & 692 & - & - & - & - & - & - & 330 & 362 & 692 \\
\hline 1934 & 1 & - & - & 1 & - & - & - & 1 & 15 & - & 15 & 349 & 407 & 756 & - & - & - & - & - & - & 349 & 407 & 756 & - & - & - & - & - & - & 349 & 407 & 756 \\
\hline 1935 & 1 & - & - & 1 & - & - & - & 1 & 19 & 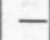 & 19 & 369 & 435 & 804 & - & - & - & - & - & - & 369 & 435 & 804 & - & - & - & - & - & - & 369 & 435 & 804 \\
\hline 1936 & 1 & - & - & 1 & - & - & - & 1 & 18 & 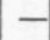 & 18 & 354 & 406 & 760 & - & - & - & - & - & - & 354 & 406 & 760 & - & - & - & - & - & - & 354 & 406 & 760 \\
\hline 1937 & 1 & - & - & 1 & - & - & $1-$ & 1 & 19 & & 19 & 381 & 436 & 817 & - & - & - & - & - & - & 381 & 436 & 817 & $1-$ & - & - & - & - & - & 381 & 436 & 817 \\
\hline
\end{tabular}

1) B.L.O. = beperkt lager onderwijs; in 1930 had de eerste omzetting van G.L.O.-scholen in B.L.O.-scholen plaats. 